\title{
Room Temperature Scanning Micro-Hall Probe Microscopy Under Extremely Large Pulsed Magnetic Fields
}

\author{
Adarsh Sandhu, Hiroshi Masuda, and Ahmet Oral
}

\begin{abstract}
The versatility of a room-temperature scanning Hall probe microscope system with an integrated minicoil capable of generating pulsed magnetic fields up to $2.9 \mathrm{~T}$ was demonstrated by imaging magnetic structures on the surface of 1.4-MB floppy disks and demagnetized strontium ferrite permanent magnets. Vibration isolation between the sample and minicoil was achieved by using a combination of quartz glass plates and silicone gel layers and enabled extremely fast measurements under fields as high as $2.9 \mathrm{~T}$, without detrimental effects on a GaAs-AlGaAs micro-Hall probe sensor located at a height of $0.5 \mu \mathrm{m}$ above the sample surface.
\end{abstract}

Index Terms-Hall effect, magnetic domains, scanning Hall probe microscopy.

\section{INTRODUCTION}

A fundamental understanding of the behavior of magnetic domains in external magnetic fields is important for the development of ultrahigh-density magnetic recording media, high coercivity permanent magnets and multilayered ferromagnetic structures used for fabricating novel "spintronic" devices [1]. Magnetic force microscopy (MFM) has been extensively used for visualizing localized stray magnetic fields at the surfaces of magnetic recording media with nanometer-scale spatial resolution [2]. However, standard MFM imaging is difficult under large external bias fields due to problems related to the magnetization of cantilever tips used for sensing surface fields. Recently, we have demonstrated the complementary technique of room-temperature scanning Hall probe microscopy (RT-SHPM) to be a highly sensitive, noninvasive, and quantitative means of sensing localized stray fields at the surfaces of magnetic recording media, garnet thin films, and permanent magnets in the presence of static external magnetic fields up to 3500 Oe [3], [4] applied using permanent magnets. In principle, it would be possible to generate much larger fields by passing pulsed currents through coils, but in practice, the integration of pulse coils with the RT-SHPM system has proven to be difficult due to the adverse affects of external vibrations

Manuscript received January 8, 2003. This work was supported in part the Japanese Ministry of Education, Culture, Sports, Science and Technology, Japan, under Grant in Aid 13650354, by TUBITAK under Grant TBAG-1878, and by Bilkent University, Turkey, under the Faculty Development Grant.

A. Sandhu is with the Research Centre for Quantum Effect Electronics, Tokyo Institute of Technology, Tokyo 152-8552, Japan (e-mail: sandhu@pe.titech.ac.jp).

H. Masuda is with the Toei Kogyo, Machida 194-0035, Japan.

A. Oral is with Department of Physics, Bilkent University, 06533 Ankara,

Turkey (e-mail: ahmet@fen.bilkent.EDU.TR).

Digital Object Identifier 10.1109/TMAG.2003.816183

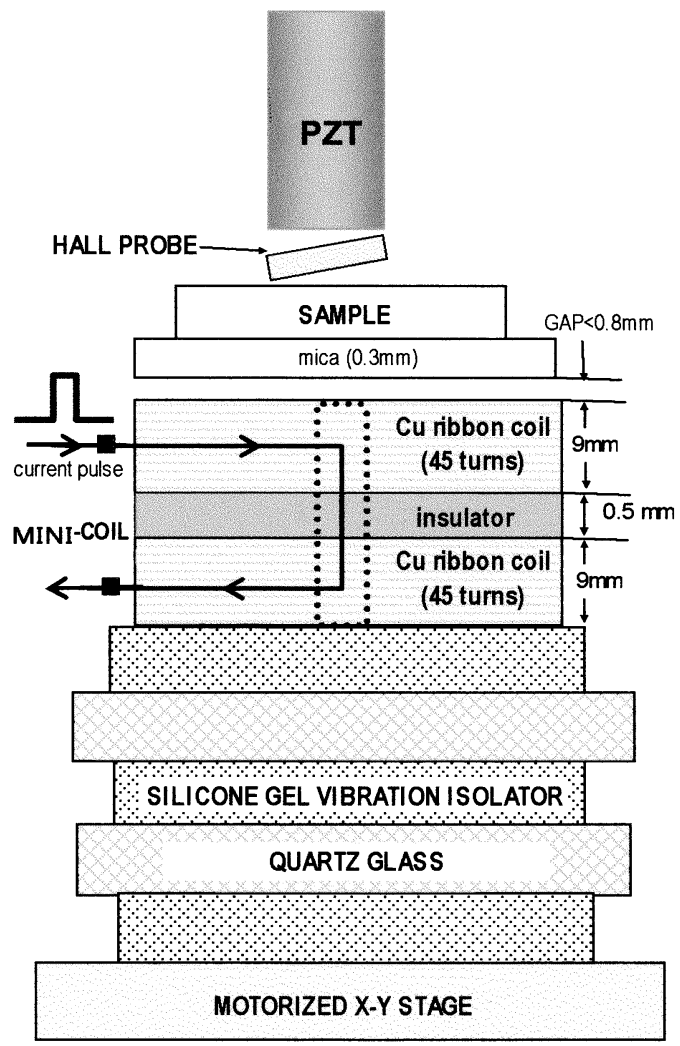

Fig. 1. Main components of RT-SHPM with an integrated 40-mm-diameter minicoil for applying large pulsed magnetic bias fields perpendicular to sample surfaces. Not to scale.

during magnetic imaging when the Hall probe is approximately $0.5 \mu \mathrm{m}$ from the sample surface.

In this paper, we report on the construction of an RT-SHPM system incorporating a unique minicoil capable of generating pulsed magnetic fields up to $2.9 \mathrm{~T}$ (width of $3 \mathrm{~ms}$ ) without damaging the Hall sensor during imaging when the Hall probe is 0.5 $\mu \mathrm{m}$ above the sample surface. The minicoil/RT-SHPM system was used for imaging the surfaces of floppy disks and strontium ferrite permanent magnets under extremely large external pulsed bias fields, thus demonstrating the feasibility of wider ranging applications of this technology.

\section{EXPERIMENT}

The main components of the minicoil and RT-SHPM [5] are shown in Fig. 1. Magnetic imaging was carried out as previously described [3] by scanning a micro-Hall probe (HP) over 


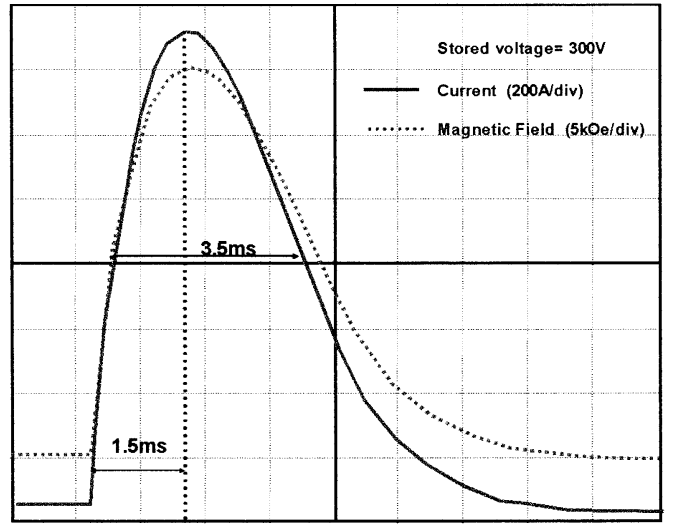

Fig. 2. Waveform of an applied current pulse and resulting magnetic field generated perpendicular to the minicoil surface.

the surface of the sample. The fabrication and characteristics of the HPs used for RT-SHPM to date have been previously reported [6]-[8]. The measurements described in this paper were carried out using a GaAs-AlGaAs 2-DEG heterostructure with an active area of $0.8 \mu \mathrm{m}^{2}$ and sensitivity of $0.04 \mathrm{GHz}$. The HP was kept at a constant height of $0.5 \mu \mathrm{m}$ above the sample surface and scanned over an area of $25 \mu \mathrm{m} \times 25 \mu \mathrm{m}$.

Pulsed magnetic fields were generated by two spiral shaped $\mathrm{Cu}$ ribbon coils (each of 45 turns, width of $9 \mathrm{~mm}$, a thickness of $200 \mu \mathrm{m}$, and a total length of $5 \mathrm{~m}$ ) wound around a common concentric core and connected in series. The $\mathrm{Cu}$ ribbons making up the coil were electrically insulated using 50- $\mu \mathrm{m}$-thick Mylar insulating film and the touching surfaces of the coils were electrically isolated by a $0.5-\mathrm{mm}$-thick insulator. The use of $\mathrm{Cu}$ ribbon instead of conventional wire coils has the advantages of a lower electrical inductance, operation at lower voltages and larger current carrying capacity in addition to an easier fabrication procedure. The coil was excited by a current pulse from a bank of electrolytic capacitors forming part of an electrical circuit that was designed to generate both polarities of pulsed magnetic fields perpendicular to the sample surface.

The distribution and magnitude of the field generated by the minicoil was measured using a commercially available, calibrated, 0.5-mm-sized Hall sensor. Fig. 2 shows the waveform of the pulsed current and corresponding magnetic field. The minicoil is capable of generating pulsed fields with full width at half maximum of $3.5 \mathrm{~ms}$ and a rise time of $1.5 \mathrm{~ms}$.

Vibration isolation of the minicoil unit and sample stage was achieved by resting the minicoil onto a structure consisting of alternate layers of a soft silicone gel tape [9] and quartz glass plates, as shown in Fig. 1. The amplitude of vibrations perpendicular to the sample surface were experimentally verified using a laser Doppler system and were found to range between 0.08 and $0.2 \mu \mathrm{m}$ for excitation pulsed currents between 200 and 1100 A. Such amplitudes of oscillations were sufficiently small to permit continuous measurements even when external pulsed fields were applied with the Hall probe at a distance of $0.5 \mu \mathrm{m}$ above the sample.

The $25 \mu \mathrm{m} \times 25 \mu \mathrm{m}$ RT-SHPM magnetic images were acquired in times ranging between 1 and $10 \mathrm{~s}$; timeframes much longer than the $\sim 3$ ms width of the pulsed bias fields.

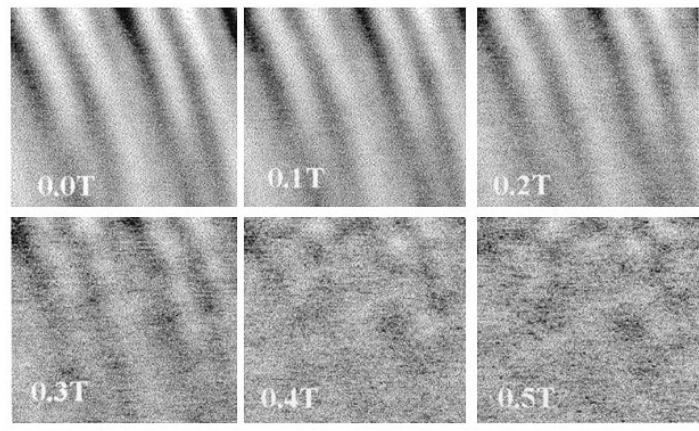

Fig. 3. Evolution of $25 \mu \mathrm{m} \times 25 \mu \mathrm{m}$ RT-SHPM images of a 1.4-MB floppy disk under increasing external pulsed bias fields.
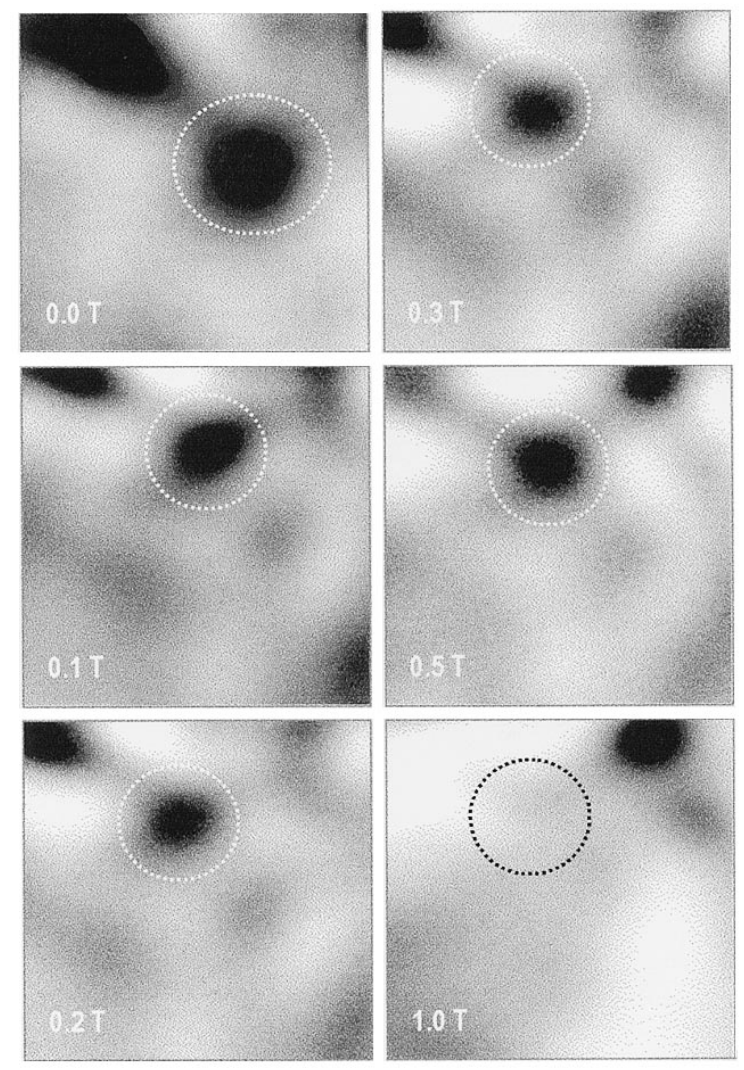

Fig. 4. $25 \mu \mathrm{m} \times 25 \mu \mathrm{m}$ images of domain dynamics of a $200-\mu \mathrm{m}$-thick demagnetized strontium ferrite sample with the pulsed field applied parallel to the easy axis. The broken circles show regions of domain movement.

\section{RESULTS AND DiscUSSION}

The minicoil/RT-SHPM system was used for imaging the surfaces of magnetic recording media and permanent magnets.

Fig. 3 shows typical $25 \mu \mathrm{m} \times 25 \mu \mathrm{m}$ images of a 1.4-MB written floppy disk under increasing perpendicular pulsed magnetic fields. The measurements were carried out immediately after applying the pulsed fields. The black and white regions in the images represent magnetizations into and out of the plane of the paper. The initial distinct magnetic transitions are seen to deform and coalesce into island-like regions at fields approaching the saturation field of $5000 \mathrm{Oe}$, as verified by VSM measurements. The stray surface fields measured by the RT-SHPM decreased from $\pm 60 \mathrm{G}$ to less than $\pm 10 \mathrm{G}$ as the external pulsed field were increased from 0 to $0.5 \mathrm{~T}$. 


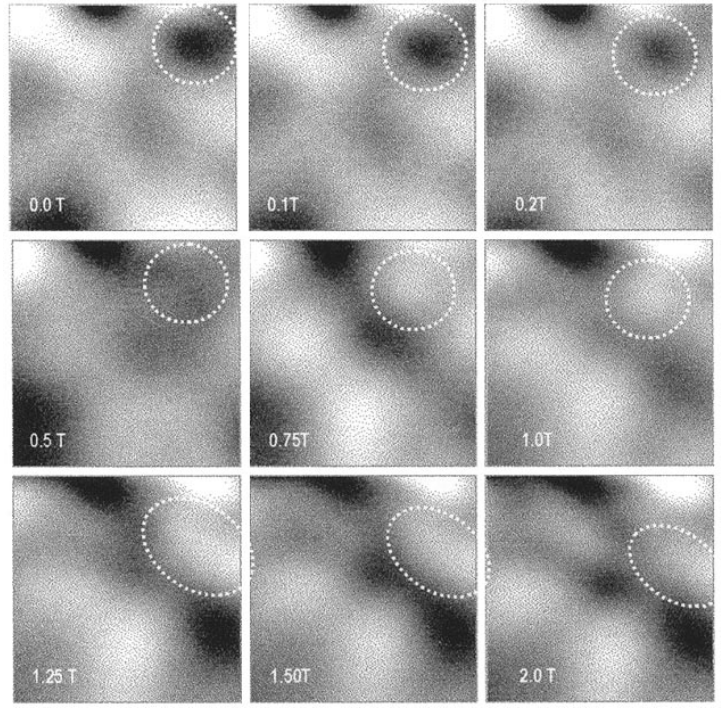

Fig. 5. $25 \mu \mathrm{m} \times 25 \mu \mathrm{m}$ images of domain dynamics of a $210-\mu \mathrm{m}$-thick demagnetized strontium ferrite sample with the pulsed field applied parallel to the hard axis, that is, normal to the image surface.

Fig. 4 shows typical $25 \mu \mathrm{m} \times 25 \mu \mathrm{m}$ images (easy axis normal to the image surface) of the variation of stray surface fields with increasing pulsed magnetic fields applied parallel to the easy axis of a $200-\mu \mathrm{m}$-thick demagnetized strontium ferrite permanent magnet, measuring $5 \mathrm{~mm} \times 5 \mathrm{~mm}$. The sample was prepared by a process involving sintering and had grain sizes ranging between 2 and $10 \mu \mathrm{m}$ [10]. The RT-SHPM images show regions on the sample with magnetization perpendicular to its surface due to the existence of clusters of multidomain grains. Application of external pulsed fields is seen to produce domain movement including reversal of certain domains at the highest fields as exemplified by the broken lines. The gray scale of the images varied between $\pm 200 \mathrm{G}$ to $\pm 140 \mathrm{G}$ as the pulsed field was increased from $0-1.0 \mathrm{~T}$.

Fig. 5 shows $25 \mu \mathrm{m} \times 25 \mu \mathrm{m}$ images (hard axis normal to the surface) of a $210-\mu \mathrm{m}$-thick demagnetized strontium ferrite permanent magnet sample measuring $5 \mathrm{~mm} \times 5 \mathrm{~mm}$ with increasing pulsed magnetic fields applied parallel to the hard axis.
Again, domain wall movement is observed. The gray scale of the images varied between $\pm 35 \mathrm{G}$ and $\pm 61 \mathrm{G}$ as the pulsed field was increased from 0 to $2.0 \mathrm{~T}$.

\section{CONCLUSION}

We described the design and construction of a minicoil unit capable of producing pulsed magnetic fields up to $2.5 \mathrm{~T}$ for integration with a room temperature scanning Hall probe microscopy system. The combined minicoil/RT-SHPM system was demonstrated to be a valuable instrument for the direct, quantitative, and noninvasive observation of localized stray magnetic fields at the surfaces of magnetic recording media and ferromagnetic materials in the presence of large external bias fields.

\section{REFERENCES}

[1] G. A. Hubert and R. Schafer, Magnet Domains. Berlin, Germany: Springer-Verlag, 1998.

[2] U. Hartmann, "Magnetic force microscopy," Annu. Rev. Mater. Sci., vol. 29, pp. 53-87, 1999.

[3] A. Sandhu, H. Masuda, A. Oral, and S. J. Bending, "Room temperature imaging of magnetic domains in storage media in the presence of external magnetic fields by scanning hall probe microscopy," in Proc. 8th Asia Pacific Phys. Conf., 2000, pp. 627-629.

[4] - "Room temperature sub-micron magnetic imaging by scanning hall probe microscopy," Jpn. J. Appl. Phys., vol. 40, pp. 4321-4324, 2001.

[5] Room Temperature Scanning Hall Probe Microscope, NanoMagnetics Instruments Ltd., Oxford, U.K., http://www.nanomagnetics-inst.com.

[6] A. Sandhu, H. Masuda, A. Oral, and S. J. Bending, "Direct magnetic imaging og ferromagnetic domain structures by room temperature scanning hall probe microscopy using a bismuth micro-hall probe," Jpn. J. Appl. Phys., vol. 40, pp. L524-L527, 2001.

[7] A. Sandhu, H. Masuda, K. Kurosawa, A. Oral, and S. J. Bending, "Bismuth Nano-Hall probes fabricated by focused ion beam milling for direct magnetic imaging by room temperature scanning Hall probe microscopy," Electron. Lett., vol. 37, no. 22, pp. 1335-1336, 2001.

[8] A. Oral, M. Kaval, M. Dede, H. Masuda, A. Okamoto, I. Shibasaki, and A. Sandhu, "RT-SHPM imaging of Garnet films using new high performance InSb sensors," IEEE Trans. Magn., vol. 38, pp. 2438-2440, Sept. 2002.

[9] Aplha-Gel thin tapes, Geltech Co. Ltd., Tokyo.

[10] A. Sandhu, N. Iida, H. Masuda, A. Oral, and S. J. Bending, "Direct and real time observation of sub-micron domain dynamics in magnetically biased strontium ferrite permanent magnets by RT-SHPM," in Proc. Mat. Res. Soc. Symp., vol. 674, 2001, pp. U3.2.1-U3.2.6. 